\title{
Epstein-Barr virus in the cerebrospinal fluid of HIV-positive patients. Observational cross- sectional study
}

\author{
Ruxandra V Moroti-Constantinescu ${ }^{1,2^{*}}$, Ioana D Olaru ${ }^{1}$, Adriana Hristea ${ }^{1,2}$, Ana-Maria Petrescu', Victoria Aramă ${ }^{1,2}$, \\ Elisabeta O Benea ${ }^{1,2}$, Raluca M Hrişcă ${ }^{1}$, Adrian Streinu-Cercel ${ }^{1,2}$, Dan Oțelea ${ }^{1}$, Dragoş Florea ${ }^{1}$ \\ From The 7th Romanian National HIV/AIDS Congress and The 2nd Central European HIV Forum \\ Sibiu, Romania. 29-31 May 2014
}

Different viruses are detected in the cerebrospinal fluid (CSF) of HIV-positive patients, with controversial implications in central nervous system (CNS) impairment. Objectives: to evaluate the presence, frequency and associations of Epstein-Barr virus (EB) in the CSF in HIV-positive patients.

We retrospectively analyzed CSF samples from HIVpositive adult patients ( $\geq 18$ years old), with or without CNS impairment, collected between Oct 2011 and Oct 2012 in a Romanian tertiary infectious diseases hospital.

We performed a multiplex PCR coupled with electrospray ionization - time-of-flight mass spectrometry (Abbott Molecular) which can simultaneously detect: herpesviruses (1-5 and 8), polyomaviruses, enteroviruses, adenoviruses and parvoviruses.

The patients were characterized based on the immunological and virological HIV status, neurological findings (including neuroimagery and CSF exam) and comorbidities.

A number of 55 patients were analyzed, with a mean age of 33.4 years (31.5 median) and a male:female ratio of 1.9:1. The CD4 count had a mean of 32 (75 median and $\mathrm{IQR}=225)$.

The most frequently detected virus was EB in 20/55 cases.

The EB-positive subgroup had a similar mean age of 33.5 years (median of 31.0) but a different male:female ratio, of 1.2:1. The CD4 count had a mean of 105.7 (59.5 median and IQR=156).

The following analyses refer to the EB-positive group: $17 / 20$ patients had neurologic impairment. Imagery was

${ }^{1}$ Carol Davila University of Medicine and Pharmacy, Bucharest, Romania Full list of author information is available at the end of the article performed in 15 cases and was normal in 5 . CSF cellularity had a median of $2 / \mathrm{cmm}, \mathrm{IQR}=10$, CSF-glucose a median of $48.0 \mathrm{mg} / \mathrm{dL}, \mathrm{IQR}=34$ and CSF-protein a median of $55.5 \mathrm{mg} / \mathrm{dL}, \mathrm{IQR}=49.5$.

In $8 / 20$ cases EB was found as singular agent in the CSF and in $12 / 20$ it was associated with other microorganisms: other herpesviruses (3 cases), JC virus (4 cases), Mycobacterium tuberculosis (2 cases), Cryptococcus neoformans (2 cases) and Toxoplasma gondii (one case).

The CSF-HIV viral load was available in $12 / 20$ cases, being detectable in 10 cases.

Regarding the neurological events (totalizing the neurological signs/symptoms, CSF exam and imagery), EB was probably causal in $4 / 20$ cases, possibly causal in another $4 / 20$ cases and was a bystander in 12/20 (3 cases with no impairment and 9 cases in which the impairments were strongly attributable to another germ).

EB is the most frequent agent found in the CSF (40\% of HIV-positive cases), mainly in women. It can be mono-detected but it especially appears in multiple associations. In more than half of the cases EB acts as an innocent bystander.

\section{Authors' details}

${ }^{1}$ Carol Davila University of Medicine and Pharmacy, Bucharest, Romania. ${ }^{2}$ National Institute for Infectious Diseases "Prof. Dr. Matei Balş", Bucharest, Romania.

\section{Published: 29 May 2014}

\section{doi:10.1186/1471-2334-14-S4-O27}

Cite this article as: Moroti-Constantinescu et al: Epstein-Barr virus in the cerebrospinal fluid of HIV-positive patients. Observational crosssectional study. BMC Infectious Diseases 2014 14(Suppl 4):O27. 